\title{
Real time fatigue damage growth assessment of a composite three- stringer panel using passive thermography
}

\author{
Joseph N. Zalameda*a Eric R. Burke ${ }^{\mathrm{a}}$, Michael R. Horne ${ }^{\mathrm{b}}$, and James B. Bly ${ }^{\mathrm{c}}$ \\ ${ }^{a}$ NASA Langley Research Center Hampton, VA 23681-2199; \\ ${ }^{b}$ National Institute of Aerospace NASA Langley Research Center Hampton, VA 23681-2199 \\ ${ }^{c}$ Lockheed Martin NASA Langley Research Center Hampton, VA 23681-2199
}

\begin{abstract}
Fatigue testing of advanced composite structures is critical to validate both structural designs and damage prediction models. In-situ inspection methods are necessary to track damage onset and growth as a function of load cycles. Passive thermography is a large area, noncontact inspection technique that is used to detect composite damage onset and growth in real time as a function of fatigue cycles. The thermal images are acquired in synchronicity to the applied compressive load using a dual infrared camera acquisition system for full (front and back) coverage. Image processing algorithms are investigated to increase defect contrast areas. The thermal results are compared to non-immersion ultrasound inspections and acoustic emission data.
\end{abstract}

Keywords: Thermal nondestructive evaluation, fatigue damage detection, aerospace composite inspection, acoustic emission, non-immersion ultrasound

\section{INTRODUCTION}

Aerospace components are commonly made with flat or curved panels with bonded stiffeners. Of interest is the debonding mechanism between the composite panel and stiffener. Progressive damage analysis (PDA) of advanced composites studies the various damage modes such as fiber fracture, matrix cracks or fiber kinking [1]. The PDA models predict damage growth as a function of applied cyclic loading and therefore validation of these models are crucial. Structural load testing is used to validate the PDA models as well as the test article designs [1,2]. Previous work has focused on testing single stringer panels $[3,4]$ and in a building block approach more realistic three-stringer panels with barely visible impact damage (BVID) has recently been tested. For a given composite design, understanding how damage initiates and grows improves confidence in predicting fatigue life.

Inspection methods are required to determine damage initiation and growth in advanced composite structures during controlled load testing. The significant damage modes for composite structures are delamination/debonding, fiber breaks, and through-the-thickness matrix cracking. In-situ inspection methods are necessary because there is potential to grow the damage if the test article is removed for inspection. In addition to removing potential handling induced damage, the in-situ inspection techniques are more time efficient. In this work, nondestructive evaluation (NDE) is used to detect the onset and growth of damage during controlled fatigue loading. The inspection is performed while the structure is on the load frame. The in-situ inspection techniques used were passive thermography, non-immersion ultrasound, fiber optic strain sensing, and acoustic emission. Both the passive thermography and acoustic emission techniques provide real time inspection during fatigue loading.

Passive thermography has been shown to be an effective technique for detecting damage in composite structures during load testing because the inspection is non-intrusive [5]. This method detects and tracks damage progression in real time as dynamic loading is applied. The loading will cause damaged areas to rub or clap together, thus providing frictional heating that is detected with an infrared camera. The passive technique can provide a real-time indication of damage formation and growth. This information is useful to determine when to stop the loading. This allows for periodic

*joseph.n.zalameda@nasa.gov; phone 1 757-864-4793; fax 1 757-864-4914; http://nde.larc.nasa.gov 
inspections using non-immersion ultrasound [6]. In this paper, the thermography results are compared to non-immersion ultrasonic inspections. The thermography results are also compared to acoustic emission.

\section{SAMPLE DESCRIPTION}

The hat stiffened composite panel flat side, stringer side before instrumentation, and stringer side after instrumentation are shown in Figures 1a, 1b, and 1c respectively. The panel skin is 17 plies with a thickness of $0.32 \mathrm{~cm}$. The hat flange is 11 plies with a thickness of $0.21 \mathrm{~cm}$. BVID was placed at 4 locations (I1, M1, I2, M2) on the panel skin between the hat stiffeners. The BVID was used to simulate existing delamination damage and serves as damage growth sites. Cyclic compressive loads were applied from $-20,000$ pounds up to $-150,000$ pounds at $2 \mathrm{~Hz}$. The load was applied from the bottom while the top was held stationary. Passive thermography was used to track the damage during loading on both the flat and stringer sides. Shown in the far right image of Figure 1c are the locations of the acoustic emission sensors (circled) and fiber optic strain sensors. The fiber optic sensors were used to detect static loads during compliance testing. The acoustic emission sensors were used for real time detection of acoustic events indicating damage growth.

(a) Flat Side

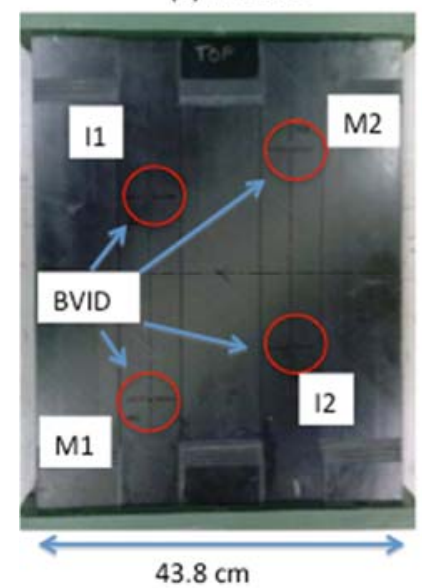

(b) Stringer Side

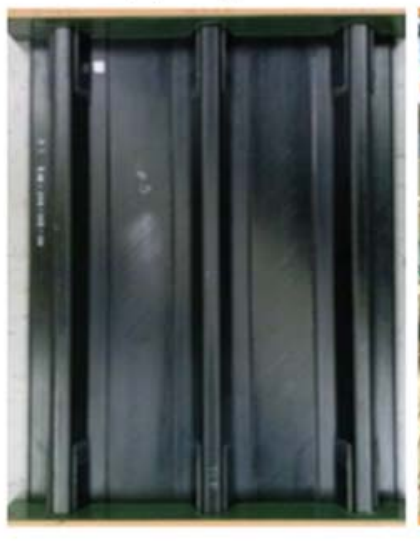

(c) Instrumented Panel

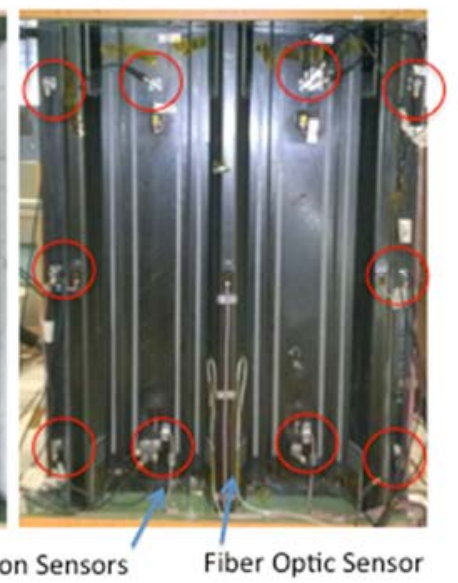

Figure 1. Three-stringer hat stiffened panel used for fatigue load testing.

\section{THERMOGRAPHY SETUP}

The thermal measurement setup is shown in Figure 2. The basic system consists of two infrared (IR) cameras operating in the 3-5 micrometer infrared band and an image data acquisition computer. The infrared cameras were configured

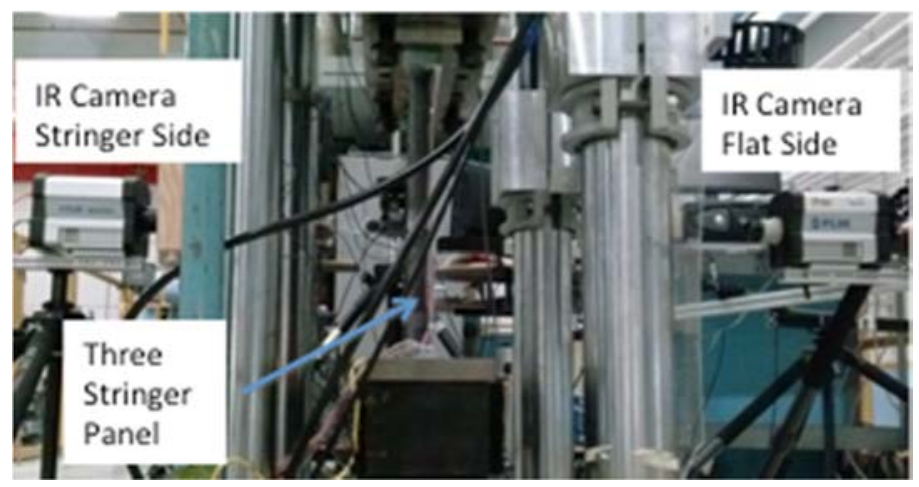

Figure 2. Three-stringer panel setup for passive thermography inspection of both flat and stringer sides. 
with $25 \mathrm{~mm}$ germanium optics. The focal plane array sizes for the cameras were from $640 \times 512$ to $1024 \times 1204$. The passive inspection captured the thermal variations during the fatigue loading. The setup required a Plexiglas ${ }^{\circledR}$ shield to contain fragments if ultimate failure occurred. The IR cameras were located behind the shield with the lens positioned in front of a viewing hole. The added benefit of the shield is it also filters out spurious IR background sources. The cameras were triggered externally using the load signal from the test machine. The sinusoidal load signal was offset corrected and the zero crossover points were used to produce a TTL trigger signal. The trigger signal was converted to a series of pulses using a pulse generator. The infrared cameras were operated at a frequency range of $16 \mathrm{Ho} 90 \mathrm{~Hz}$ depending on acquisition time duration. Higher frequencies were used to capture the damage progression as the panel approached ultimate failure. The image data are displayed in real time and stored directly to a hard drive with minimal frames dropped. This provides the capability to acquire frames for an extended period of time (hours if required).

\section{PASSIVE INSPECTION RESULTS}

\subsection{Real Time Inspection Data}

The passive inspection was performed without application of external heat. Any detectable heating was a result of thermoelastic heating, matrix cracks rubbing or delamination/disbond interfaces clapping or rubbing due to the applied load. Thermal data were both acquired and displayed in real time during cyclic loading. The infrared cameras were triggered simultaneously from the applied fatigue load signal. The real time display was used to allow the operator to watch the damage growth. If the heating area or intensity increased suddenly, then the applied load was stopped for detailed inspection using non-immersion ultrasound. This strategy allowed for controlled damage growth as a function of applied load. Example raw temperature images are shown in Figure 3. The raw unprocessed real time temperature images, shown as a function of remaining fatigue life, provide good contrast to track the increased areas of heating due to damage growth. The early load data as shown in Figure 3 for the $31.6 \%$ remaining life fatigue image does not show significant heating and therefore reveals minimal or no damage growth. Processing the images using an image

Flat Side

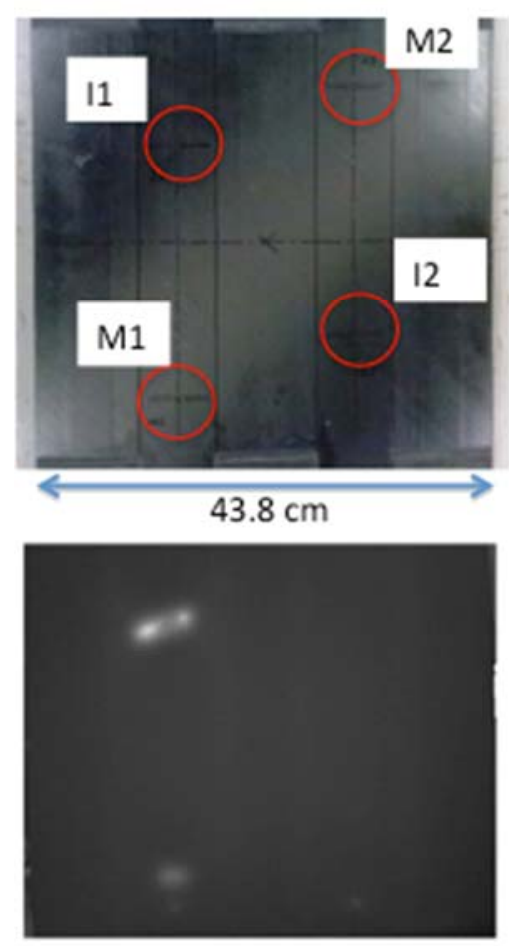

$98.6 \%$ of Life
$31.6 \%$ of Life
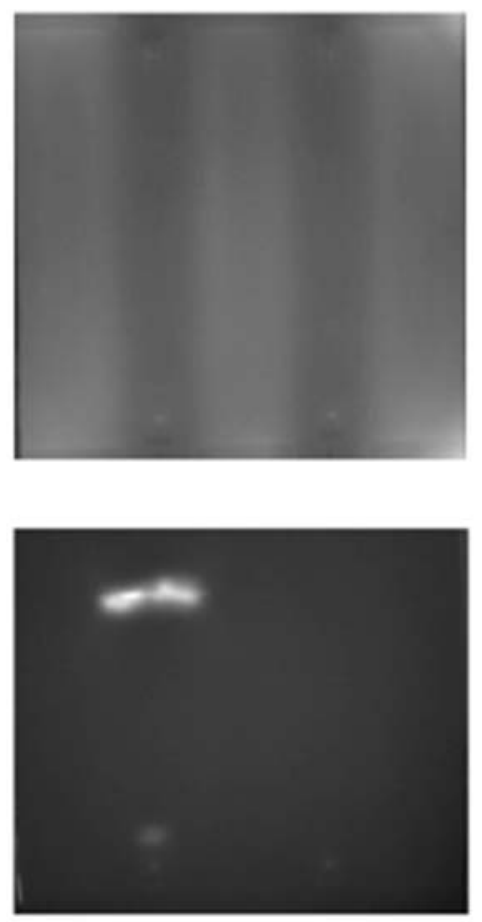

$99.2 \%$ of Life
$97.3 \%$ of Life
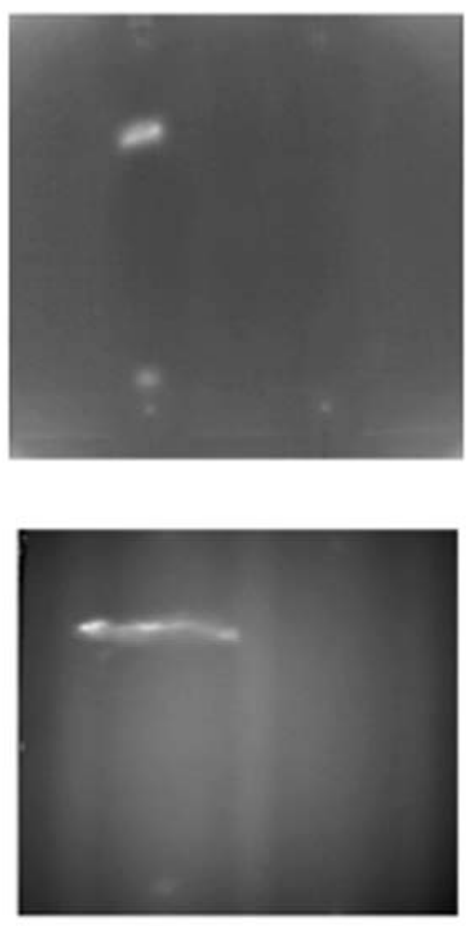

$99.9 \%$ of Life

Figure 3. Flat side passive thermography unprocessed raw temperature images as a function of remaining fatigue life. 
differencing technique [7] allows for improved contrast of the impact areas. This processing technique can be easily implemented in real time and involves only a delayed image subtraction. A moving buffer is required in the acquisition software. This delayed subtraction is given as:

$$
\text { Difference Image }=\text { Acquired Image }[i]-\text { Previous Image }[i-k / 2]
$$

where $k=$ number of images per cycle and $i$ is the current acquired image number. Dividing $k$ by 2 insures the maximum and minimum temperature images are subtracted, within a cycle, and this image provides the optimal contrast of the damaged areas. The difference images between the maximum and minimum temperature images for a given cycle are shown in Figure 4. The subtracted images reveal the damaged areas in greater contrast as compared to the raw unprocessed temperature images shown previously in Figure 3. The impact I1 is shown to clearly grow as a function $\%$ remaining life, and therefore real time difference processing is ideal for display during loading.

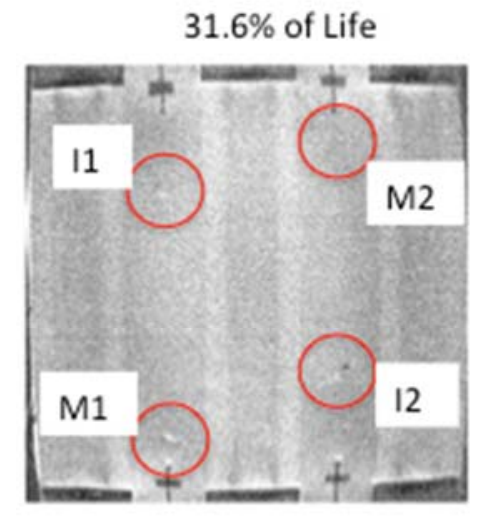

$99.2 \%$ of Life
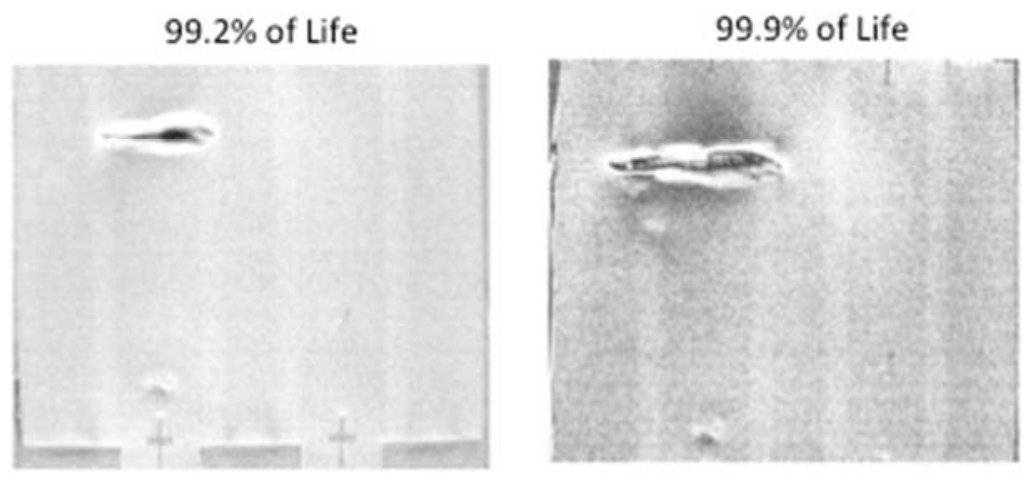

$98.6 \%$ of Life
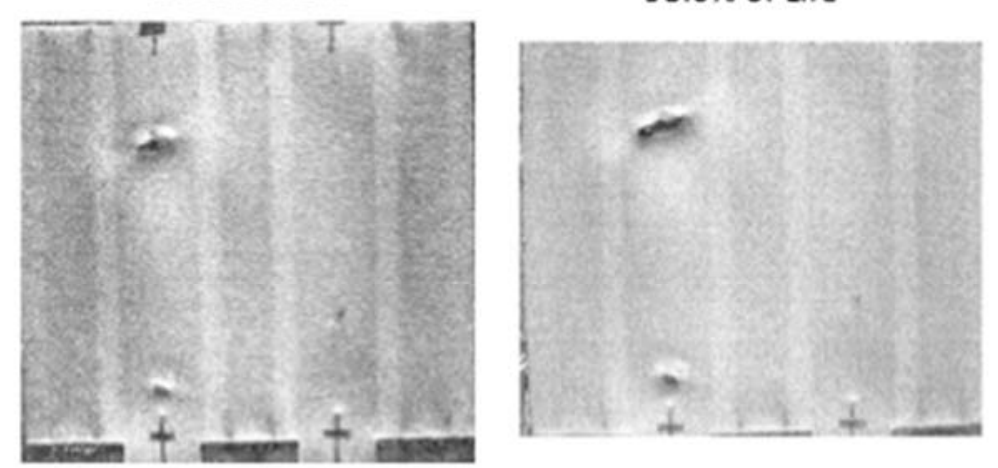

Figure 4. Difference thermography images of damage growth as a function of remaining fatigue life.

\subsection{Processing Thermography Inspection Data}

The image data are stored directly to a hard drive for processing. This provided the capability to acquire frames for an extended period of time (hours) if necessary. The stored data was subsequently analyzed to track the temperature increases in more detail. The temperature response was generated by averaging the temperature values (counts) over the panel. The average temperature and applied load are plotted in Figure 5 where the applied compressional loading increases the overall temperature and release of loading decreases the temperature. This is attributed primarily to the thermoelastic effect. For each cycle, the maximum and minimum temperature images were selected and subtracted. The difference images were then averaged over a given number of load cycles (typically around 50 cycles). Examples of the processed passive inspection results, showing detected damage, are shown in Figure 6 along with comparisons to nonimmersion ultrasonic inspection results. Frictional forces or clapping of damaged areas during loading produce 
temperature changes that allow the damaged areas to be detected. The processed thermography inspection images show good agreement to the ultrasonic inspection results.

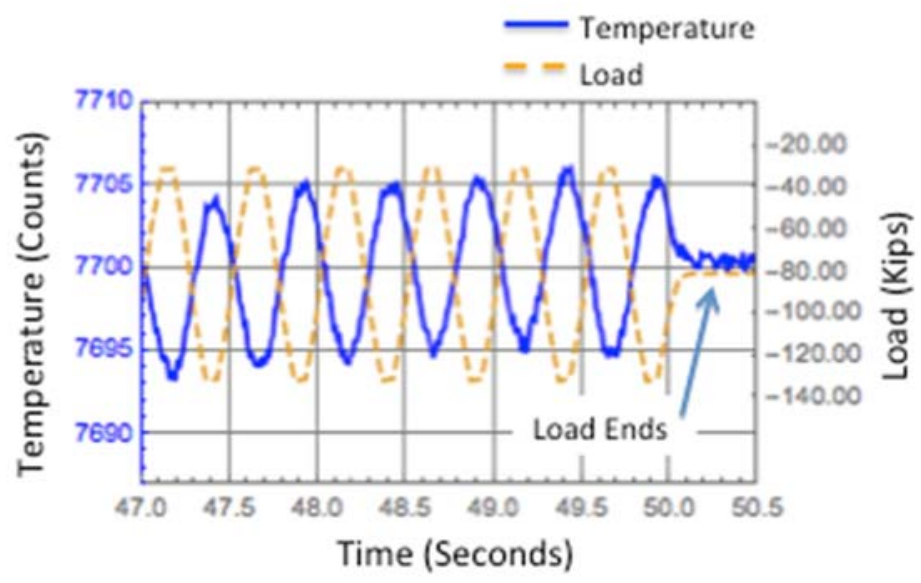

Figure 5. Averaged temperature over panel surface and applied load.

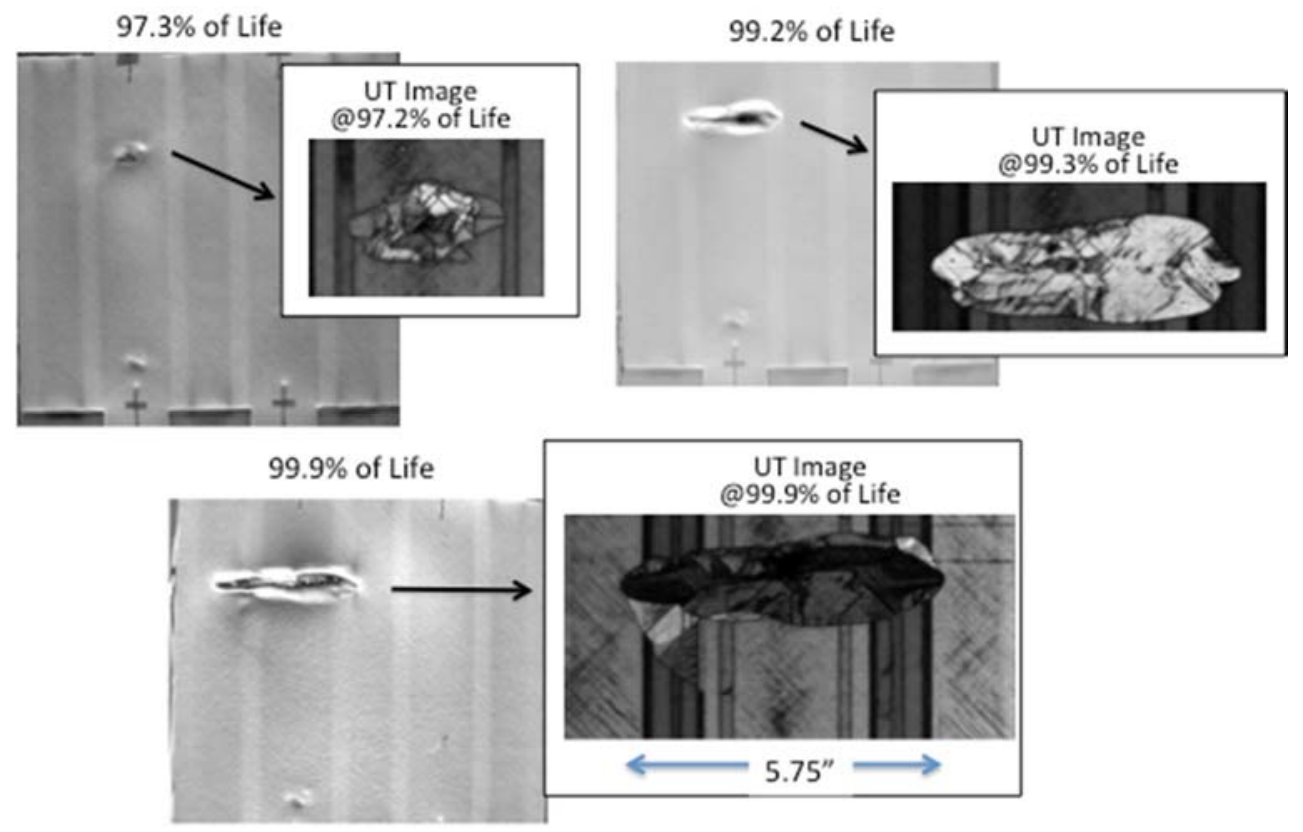

Figure 6. Comparison of processed thermography images to ultrasonic inspection results.

\subsection{Estimation of Damage Depth}

Of interest during loading is the formation of damage at multiple interfaces within the composite. In addition to the size of the damage growth, rapid damage formation at multiple layers within the composite can be a good indicator of pending ultimate failure. The applied load generates heating at the damage locations within the sample. The heating produces a strongly damped periodic thermal response that can be measured at the surface where the attenuation is estimated by:

$$
\text { attenuation }=\operatorname{Exp}\left[\frac{-z}{p}\right] \quad \text { with } \quad p=\left(\frac{\text { diff }}{\pi * \text { Freq }}\right)^{1 / 2} .
$$


The defect depth is given as $z$ and $p$ is the thermal diffusion length. The depth $z$ is related to the phase delay and thermal diffusion length $p$ as:

$$
z=\text { phase delay } * p .
$$

The values of thermal diffusivity, diff, is estimated to be $0.0045 \mathrm{~cm}^{2} / \mathrm{sec}[8]$ and the frequency Freq $=2.0 \mathrm{~Hz}$ (fatigue frequency). The attenuation and phase delay are both plotted as a function of defect depth in Figure 7. Equation (2) provides a linear estimate of the phase difference between the temperature at the defect location (depth $\mathrm{z}$ ) versus the measured surface temperature response. For near surface damage the phase delay will be small whereas for deeper defects the phase delay will be greater. Because the periodic thermal responses are highly damped, the measured defect depth is limited using phase, especially since the fatigue loading frequency is fixed at $2 \mathrm{~Hz}$. To address this limitation, 2 infrared cameras were used to capture the thermal response on both the flat and stringer sides.

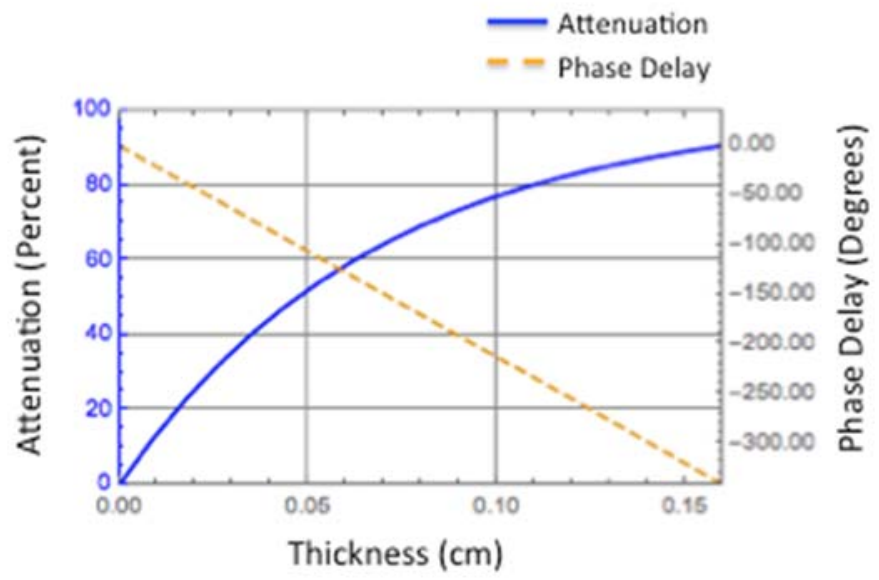

Figure 7. Comparison of attenuation and phase delay as a function of thickness.

The phase and magnitude images are calculated by taking the Fourier transform, pixel by pixel, of an image data cube $[9,10]$. The magnitude and phase images (representing 10 load cycles) at the frequency of loading $(2 \mathrm{~Hz})$ of both flat and stringer sides are shown in Figure 8. These images represent damage to within several cycles before ultimate failure. The phase delay image is used to estimate damage depth using equation 2 . The phase delay is determined by subtracting the corrected loading phase with the phase within the image. The loading phase is corrected by 180 degrees and this defines the surface phase temperature response (confirmed by Figure 5). Defects at the surface will have a phase delay of zero and deeper defects will have an increasing phase delay. This is shown in Figure 9 where the ultrasonic throughthe-thickness results are compared to the thermal phase delay image for defect I1. There appears to be good qualitative agreement for the near surface defects to ultrasonic images a and $\mathrm{b}$. The ultrasonic inspection results for $\mathrm{c}$ and $\mathrm{d}$ are barely detectable in the thermal phase image since the periodic thermal response is highly damped for deeper defects.

\subsection{Comparison to Acoustic Emission}

Fourteen acoustic emission sensors were bonded to the composite panel on the stringer side. They were connected to a data acquisition computer using pre-amplifiers and filters for capture of acoustic data. The acoustic emission computer also captured the load for synchronization. Acoustic emission systems collect structure-borne sound, typically not audible, in the ultrasonic frequency band (approx. 50 to $500 \mathrm{kHz}$ ), generated by dynamic displacements such as damage initiation and growth at picometer scales [11]. Pre-cursors to failure are monitored in real-time. Signals from the distributed array of sensors are acquired simultaneously when any one sensor threshold triggers. The signal energy, SE, of an acoustic signal is given as:

$$
S E=\sum_{i=1}^{n} V_{i}^{2} \Delta t
$$




\section{Flat Side}

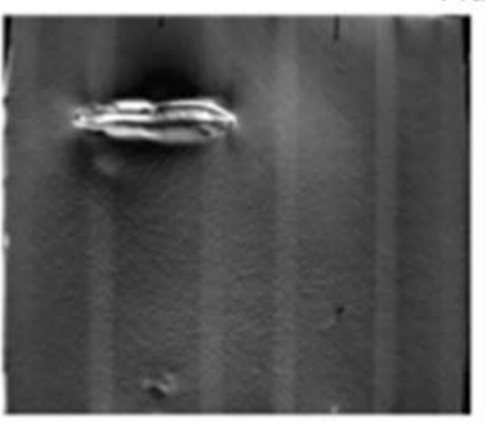

Magnitude

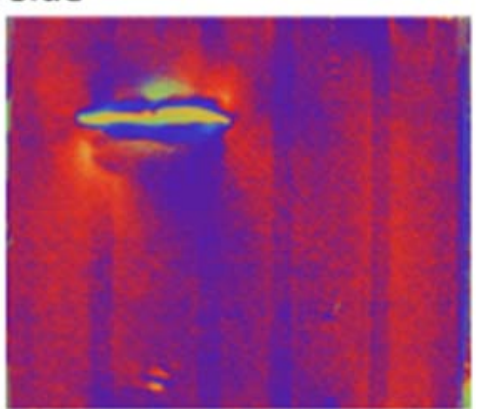

Phase

Stringer Side

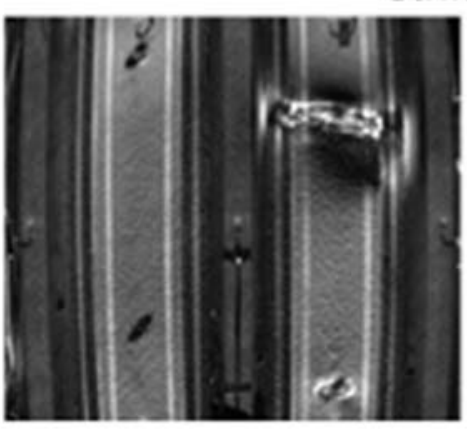

Magnitude

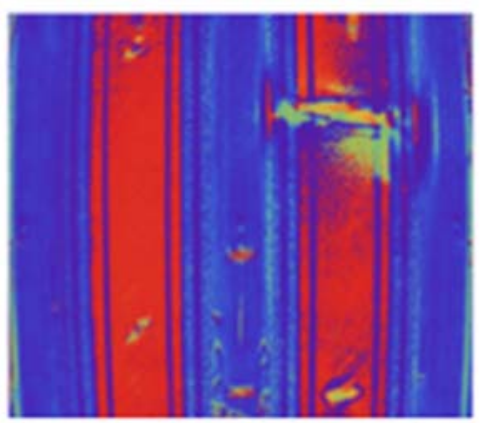

Phase

Figure 8. Magnitude and phase images (at $2 \mathrm{~Hz}$ ) of both the flat and stringer sides.

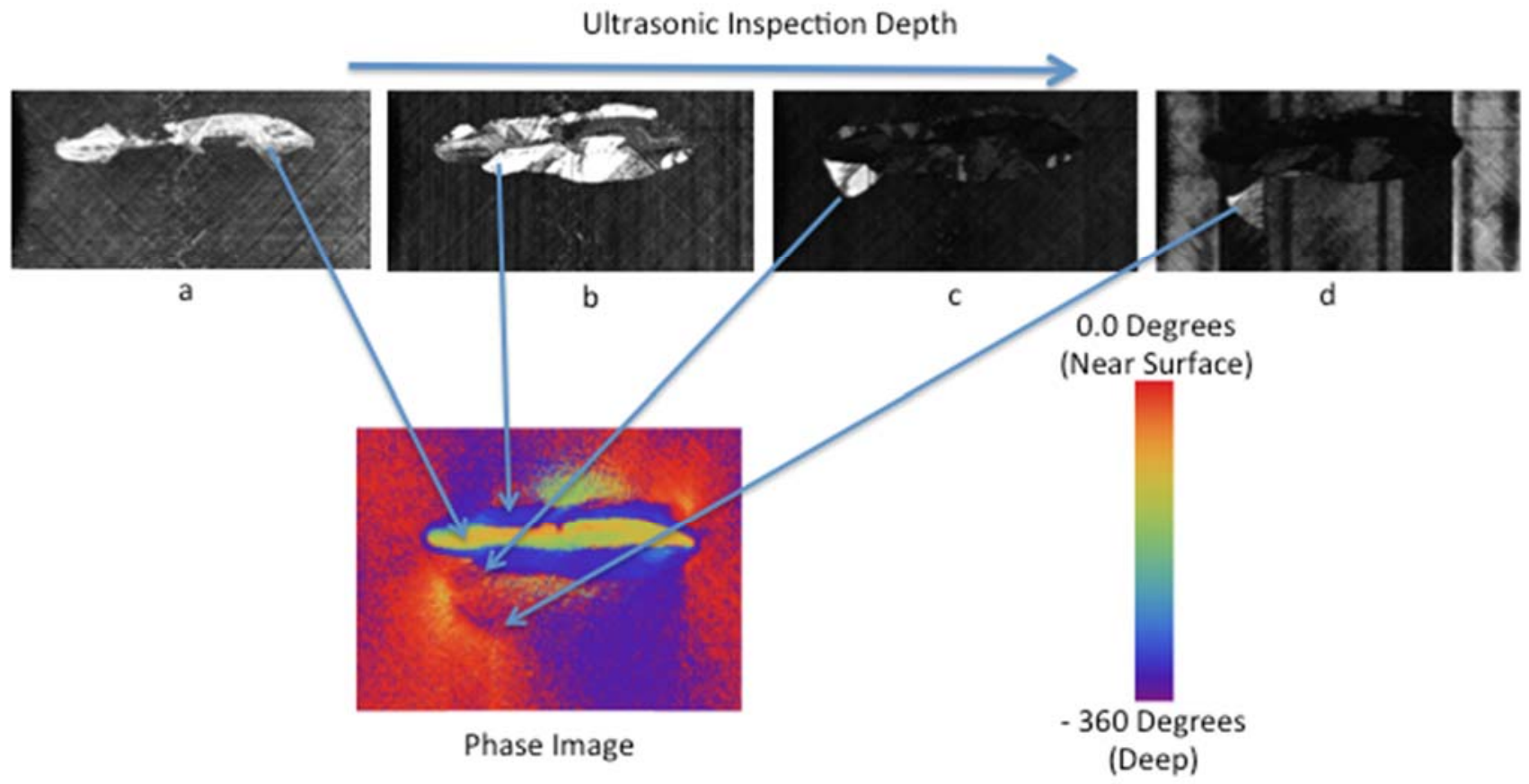

Figure 9. Comparison of phase image to ultrasonic inspections showing damage at multiple interfaces. 
where $V_{i}$ is the signal voltage, $i$ is the time reference point, $n$ is the number of time points in the signal, $\Delta \mathrm{t}$ is the sampling time per point. The total event energy is calculated by summing the $S E$ values for all the acoustic emission sensors (Figure 10). Also in Figure 10 is the averaged measured temperature over defect I1, calculated from the thermal camera image data. Trends of the energy over time, or released thermal energy perhaps can be used to identify and track damage development. For example, the release of the acoustic energy can be used to trigger the infrared camera acquisition [12]. Sudden increases, as shown in Figure 10, of both thermal and released acoustic energy show potential to predict ultimate failure. This would allow removal of load to inspect using non-immersion ultrasound before panel loss.

\subsection{Capture of Panel Ultimate Failure}

Figure 11 shows the flat and stringer side images where the acquisition of the thermal image is timed with the catastrophic failure of the composite structure. These images can be used to determine the final failure mechanism. In this panel, the growing delamination I1 initiated a disbond between the stringer flange/skin. The growing disbond between the stringer flange/skin was final failure mechanism.

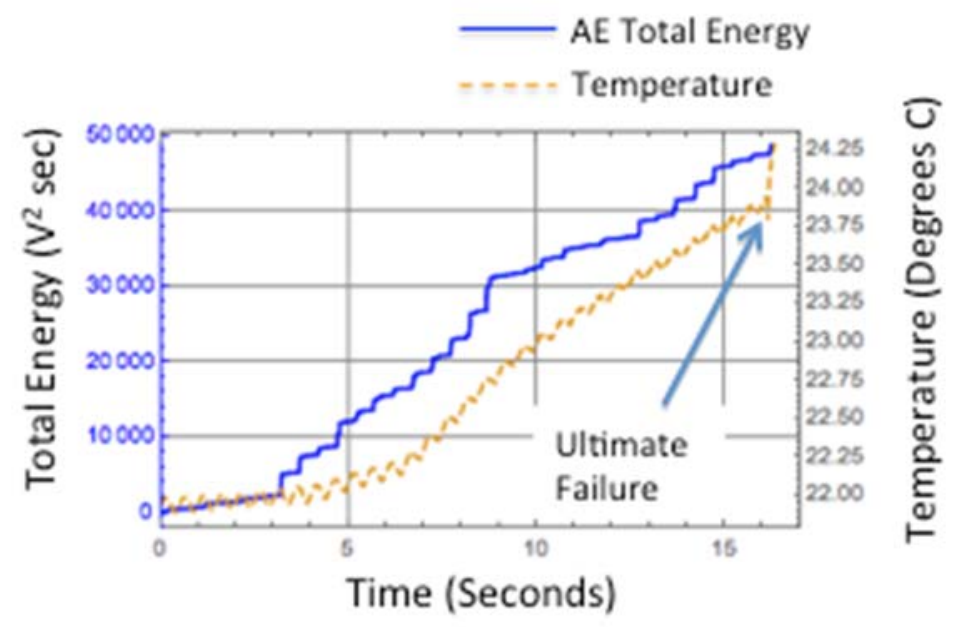

Figure 10. Comparison of total acoustic emission energy to temperature.

\section{Flat Side}

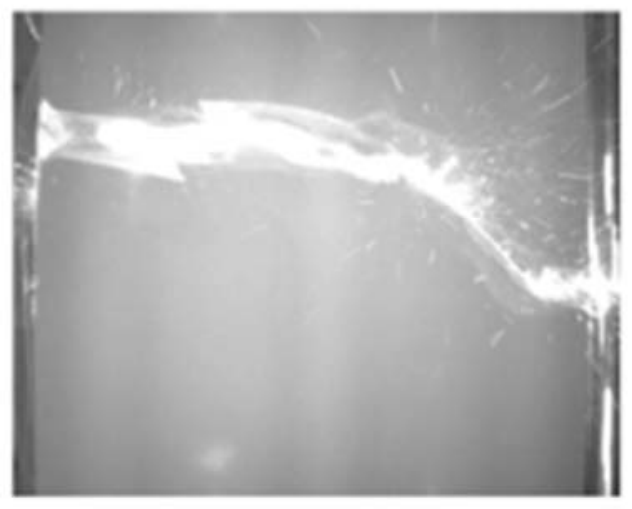

Stringer Side

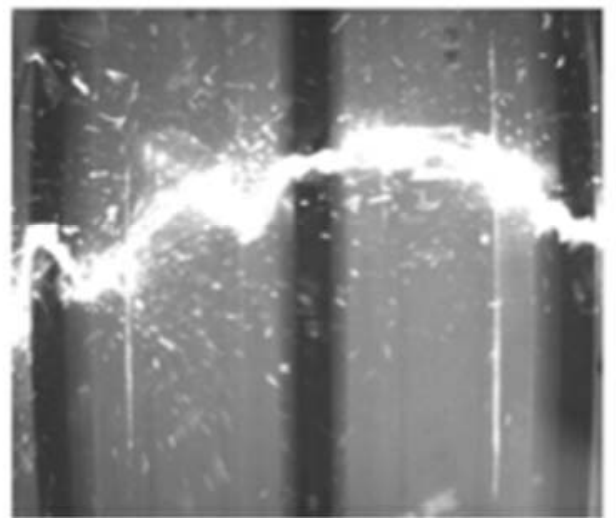

Figure 11. Thermal images showing panel ultimate failure. 


\section{CONCLUSIONS}

Passive thermography was used to track damage onset and growth on a three-stringer composite panel. A simplified processing technique, using a delayed difference technique, is presented and shown to significantly increase contrast while still providing thermal monitoring capability. This will allow for improved real time assessment of damage formation and growth during loading. In addition, passive thermography was shown to image damage at multiple interfaces (depths) by computing the phase image. Lastly, acoustic emission was compared to the temperature increase over the largest damage area (I1). Trends of the acoustic emission energy over time and/or released thermal energy, perhaps can be used to identify and track damage development, however further analysis is required to effectively combine both techniques.

\section{ACKNOWLEDGEMENTS}

The authors would like to acknowledge Wade Jackson and Christopher Wright of NASA Langley for ultrasonic inspections, sample preparation, and mounting. Also the authors would like to acknowledge William Johnston of Lockheed Martin for testing support.

\section{REFERENCES}

[1] T. K. O'Brien, "Development of a Delamination Fatigue Methodology for Composite Rotorcraft Structure", NASA Aviation Safety Technical Conference Denver, CO, October 2008.

[2] R. Krueger, "An Approach to Assess Delamination Propagation Simulation Capabilities in Commercial Finite Element Codes", NASA/TM-2008-215123, 2008.Davis, A., R., Bush, C., Harvey, J. C. and Foley, M. F., "Fresnel lenses in rear projection displays," SID Int. Symposium Digest Tech. Papers 32(1), 934-937, 2001.

[3] Chiara Bisagni, Carlos G. Dávila, Cheryl Rose, and Joseph Zalameda, "Experimental Evaluation of Damage Progression in Postbuckled Single Stiffener Composite Specimens", American Society for Composites $29^{\text {th }}$ Technical Conference Proceedings, US-Japan 16, ASTM D30, Feb. 2014.

[4] J. N. Zalameda, E. R. Burke, R. F. Parker, J. P. Seebo, C. W. Wright, and J. B. Bly, "Thermography Inspection for Early Detection of Composite Damage in Structures during Fatigue Loading", Thermosense XXXIV, edited by Douglas Burleigh, Gregory R. Stockton, Proc. of SPIE Vol. 835403 April 2012.

[5] E. G. Henneke, K. L. Reifsnider, and W. W. Stinchcomb, Journal of Metals 31(9), 11-15, 1979.

[6] P. H. Johnston, C. W. Wright, J. N. Zalameda, and J. P. Seebo, "Ultrasonic monitoring of ply crack and delamination formation in composite tube under torsion load", Ultrasonic Symposium (IUS), IEEE, pp. 595 - 598, 2010.

[7] William P. Winfree, Joseph N. Zalameda and Patricia A. Howell, "Improved flaw detection and characterization with difference thermography", Proc. SPIE 8013, 80130U April 2011.

[8] J. N. Zalameda, Journal of Composite Technology and Research 21, 98-102, 1999.

[9] X. Maldague, S. Marinetti, Pulse Phase Infrared Thermography, J. Appl. Phys. 79 [5], pp. 2694-2698, 1996.

[10] A. Dillenz, Th. Zweschper, G. Busse, Progress in ultrasound phase thermography, Proc. SPIE Vol. 4360, SPIE Bellingham, WA, pp. 574-579, 2001.

[11]Horne, M. R. "Rayleigh Wave Acoustic Emission during Crack Propagation in Steel". PhD Dissertation, Virginia Polytechnic Institute and State University. 2003.

[12] Ringermacher H. I., et al, "System and Method for Locating Failure Events in Samples Under Load", United States Patent No. 7,516,663 B2, April 14, 2009. 